\title{
Induced motion and apparent straight ahead during prolonged stimulation
}

\author{
ROBERT B. POST \\ University of California, Davis, California \\ and \\ THOMAS HECKMANN \\ University of California, Berkeley, California
}

\begin{abstract}
Induced motion (IM) of a fixation target and the location of the apparent straight ahead (ASA) were measured during and after 2 min of exposure to a moving contoured background. The magnitude of IM increased to an asymptotic level during the exposure period. Following termination of moving contours, illusory motion of the fixation target occurred first in the same direction and then in the direction opposite to that of previous IM. The change in location of ASA followed a similar time course, shifting first in the direction of background motion, reaching an asymptotic position during contour exposure, and then moving in the opposite direction after contour exposure. The similar time courses displayed by IM magnitude change and ASA position change suggest that these two phenomena may be related through a third, causal factor. The hypothesis that IM is caused by motion of ASA is disconfirmed, however, by the fact that IM persists while the ASA is nearly stationary at its asymptotic position.
\end{abstract}

Induced motion (IM) refers to the illusory motion of a visual stimulus in a direction opposite to that of the real motion of other visual detail. In 1929, Duncker suggested that changes in the relative positions of the stationary and moving visual stimuli cause the illusory motion. Since then, a number of other investigators have similarly emphasized the role of object-relative motion in the genesis of IM (see, e.g., Mack, Heuer, Fendrich, Vilardi, \& Chambers, 1985; Nakayama \& Tyler, 1978).

In contrast to explanations that emphasize the role of object-relative motion, other authors have suggested "subject-relative"' accounts. In such accounts, IM results from comparing the position of the stationary stimulus with changes in observers' egocentric orientation induced by moving stimuli. Specifically, Brosgole (1968) and others (see, e.g., Bridgeman \& Klassen, 1983) have suggested that the moving stimulus causes the location of the apparent straight ahead (ASA) of subjects to move in the direction of background motion, thereby causing illusory motion of the stationary stimulus in the opposite direction.

In contrast to the preceding classical analyses of IM, which emphasize the role of object-relative or subjectrelative principles, other authors have suggested a role for eye-movement information in the genesis of IM. Specifically, Post, Shupert, and Leibowitz (1984) have sug-

\footnotetext{
We are grateful to Marc Chaderjian and Kathy Hoyt for their comments on the manuscript. We also thank Herschel Leibowitz for serving as a pilot subject. This research was supported by University of California Grants D-1832 (to R.B.P.) and DG562 (to T.H.).

R. B. Post's mailing address is: Department of Psychology, University of California, Davis, CA 95616. T. Heckmann is associated with the School of Optometry at the University of California in Berkeley.
}

gested that IM occurs when the smooth ocular pursuit system opposes reflexive eye movements that would otherwise be elicited by the moving background stimulus. When the pursuit system is activated in order to prevent fixational loss, smooth pursuit activation is registered perceptually as motion of fixated detail (Post, in press; Post \& Leibowitz, 1985).

The present experiment examined the magnitude of IM and change in location of ASA during and after prolonged exposure to an IM display. Pilot measures in our laboratory had suggested that although IM is continuous during prolonged viewing of an IM display, there is a limit to the extent that ASA moves. This finding, if substantiated, would contradict the hypothesis that IM results from movement of the ASA. That is, IM cannot be attributed to changes in egocentric orientation if such changes, as measured by movement of the ASA, are absent during the continuous presence of IM. It was also anticipated that the results would be relevant to the hypothesis (Post, in press; Post et al., 1984) that suppression of reflexive eye movements by the pursuit system contributes to IM. This idea follows from evidence that activation of reflexive eye movements builds up only gradually after onset of a moving contoured stimulus (see review by Baloh, Yee, \& Hornrubia, 1982). If IM results from the suppression of reflexive eye movements by ocular pursuit mechanisms, there should be a buildup and asymptote in IM magnitude during prolonged stimulation as activation in the reflexive eye-movement system increases and asymptotes, and opposite activation in the ocular pursuit system builds up correspondingly to maintain fixation. Similarly, as a consequence of the position constancy signal associated 
with ocular pursuit effort, there should be an increase and asymptote in the deviation of ASA from the objective median plane in a direction opposite to that of IM.

\section{METHOD}

Three 28-34-year-old individuals, 2 males and 1 female, served as subjects. Each was mildly myopic, displaying no significant refractive error at the distance of the stimuli when tested without optical correction. Two of the subjects (T.H. and R.P.) were the authors; the third (K.H.) was naive with regard to the experimental hypotheses.

All observations were made binocularly, with the subject seated and his or her head movements restrained by means of a bite-bar. The subject was located at the center of a vertically aligned hemicylinder which served as a projection screen (radius = $48.5 \mathrm{~cm}$ ). Horizontally moving vertical stripes could be projected on the interior surface of the cylinder by a striped shadow-caster attached to a dc motor over the head of the subject. The striped display extended $95^{\circ}$ vertically and $180^{\circ}$ horizontally with each black-white stripe cycle subtending $15^{\circ}$ of visual angle. This pattern was selected to serve as an inducing stimulus because it satisfied the following criteria: (1) It generated IM during pilot measures, and (2) it was repetetive, thereby allowing prolonged stimulation without confounding the variables of stimulus separation and duration, as does a nonrepetetive inducer such as a moving rectangular frame. A laser and mirror galvanometer over the subject's head permitted projection of a spot of light on the surface of the hemicylinder in front of the subject.

In measuring relative magnitudes of IM under different stimulus conditions, several previous authors have taken advantage of the fact that orthogonal directions of IM and real motion can be combined to yield a path of apparent motion that is a vector sum of the two (see, e.g., Gogel \& MacCracken, 1979; Wallach, Bacon, \& Schulman, 1978). The present technique for measuring direction and magnitude of IM utilized this principle to continuously track changes in IM magnitude during prolonged stimulation. On each trial, the subject initially sat in darkness and indicated to the experimenter when he or she was ready for the trial to begin. Each trial began with the appearance, in front of the subject, of a spot of laser light, which the subject was instructed to fixate. The fixated spot then moved upward repeatedly at a rate of $3 \mathrm{deg} / \mathrm{sec}(\mathrm{dps})$ through an extent of $15^{\circ}$. The subject tracked the spot and adjusted a rod attached to a potentiometer to match the apparent slant of the path of the moving spot. After $3 \mathrm{sec}$, the moving striped background was added to the field of view, and the subject continued to adjust the slant of the rod to match the apparent slant of the moving spot. After 2 min of continuous IM path matching, the striped background was turned off and the subject continued to fixate the laser spot in darkness and to match the apparent slant of its path for another minute. Two trials were recorded for each of the 3 subjects, one with a background velocity of $5 \mathrm{dps}$ and the other with a background velocity of $60 \mathrm{dps}$.

The technique for measuring ASA deviation from the objective median plane is fairly standard (see, e.g., Brecher, Brecher, Kommerell, Sauter, \& Sellerbeck, 1972; Skavenski, Haddad, \& Steinmen, 1972). Observers simply adjust a spot of light to correspond with ASA. In our experiment, subjects deflected a joystick to move a laser spot left or right to match the apparent location of the median sagittal plane. The subjects were exposed to the moving background for the same durations as those employed with IM measurement; the background velocities and number of trials were also the same. The order of testing ASA or IM was varied pseudorandomly. There was no vertical motion of the laser spot during ASA measurement in order to prevent the laser spot from appearing to move on a slant, which would produce ambiguity with respect to the portion of its motion path that corresponded to ASA. The use of phenomenally different, though nonetheless standard, measures of IM and ASA deviation eliminates a potential confounding element present in some previous comparisons of IM and ASA (e.g., Brosgole, 1968). Specifically, if IM is measured by canceling apparent motion with fixation target motion in the direction opposite to that of IM, and ASA is measured by moving the target in the direction opposite to that of IM, it is not at all clear that different responses are being measured.

Consecutive 5-sec samples of IM magnitude (slant from vertical) and ASA position (deviation from the objective median plane) were sampled for correlational analysis. This frequency for data representation was selected because preliminary measurements using continuous recording of IM magnitude and faster $(1 \mathrm{~Hz})$ sampling of ASA position had shown changes in both variables to be smooth and monotonic.

\section{RESULTS}

Consecutive 5-sec samples of IM magnitude obtained with a background velocity of $5 \mathrm{dps}$ are plotted for each subject in the top portion of Figure 1. The apparent motion path of the tracked spot begins slanting leftward from vertical (opposite background motion) shortly after moving background onset. Slant increases for about $60 \mathrm{sec}$ before reaching an asymptotic level. After cessation of the moving background, slant rapidly decreases and then reverses. Each subject's responses display this pattern, and the asymptotic levels of slant vary somewhat among subjects.

Consecutive 5-sec samples of ASA position during and after a background velocity of $5 \mathrm{dps}$ are plotted for each subject in the bottom portion of Figure 1. ASA positionchange displays a temporal course similar to that of the IM results given in the top half of the figure. Specifically, ASA begins to shift in the direction of background motion, and reaches an asymptotic displaced location after about $60 \mathrm{sec}$. Following cessation of the moving background, ASA location moves rapidly in the direction opposite to that of the background motion. The ASA of each subject has moved to the left of the objective midline by the end of the recording interval.

Results obtained with a 60-dps background velocity are presented in Figure 2 for individual subjects. Overall, the IM and ASA measures display a pattern highly similar to that presented in Figure 1 for the 5-dps background velocity. As is evident from a comparison of Figures 1 and 2, asymptotic IM magnitudes and ASA displacements during background motion were greater across all 3 subjects for the 60-dps stimulation.

Correlations were computed between the 5-sec samples of ASA location and IM magnitude for individual subjects separately at each stimulus velocity. Separate analyses were conducted for the results obtained before and after termination of the moving background. A summary of these correlations is provided in Table 1. For background velocities of $60 \mathrm{dps}$, IM magnitude is significantly correlated with ASA location for all subjects, both before and after stimulus termination. With 5-dps background velocity, IM magnitude and ASA location are sig- 


\section{DEG/SEC BACKGROUND MOTION}

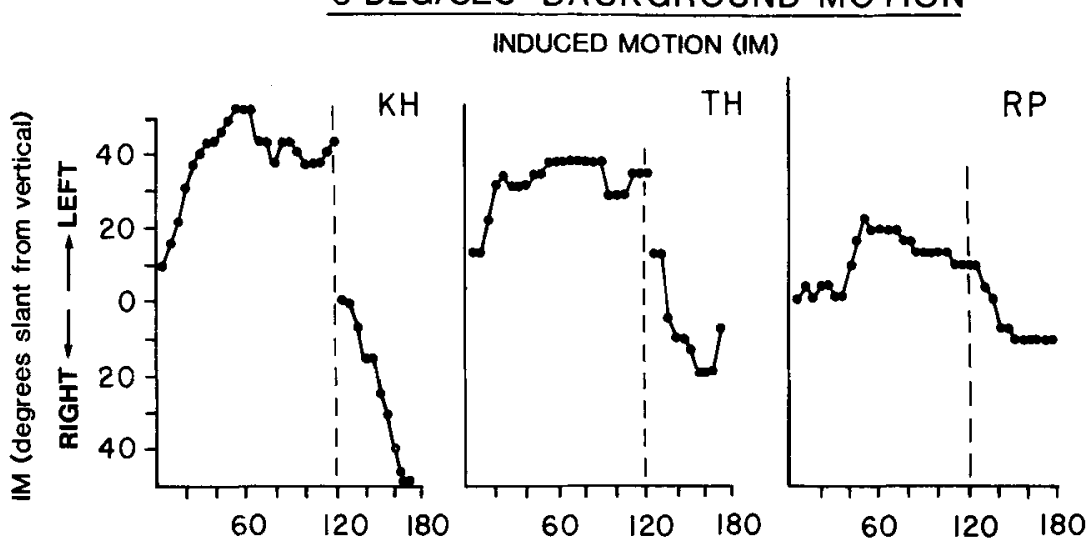

APPARENT STRAIGHT-AHEAD (ASA) POSITION

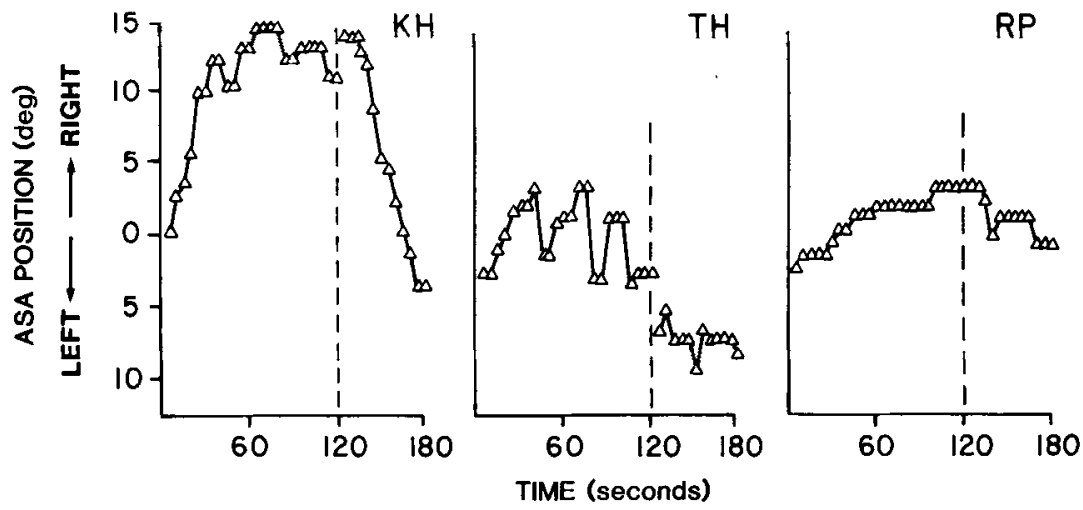

Figure 1. Top panel: Induced motion magnitude for individual subjects across 5-sec time samples during and after presentation of a 5-dps moving background. Bottom panel: Apparent straight-ahead position with respect to the objective straight ahead during the same time intervals for individual subjects. Note that, in all figures, leftward IM is plotted upward on the ordinate, while rightward ASA is plotted upward on the ordinate. These plots are therefore consistent with the direction of IM and ASA changes induced by the moving stimulus. The vertical dashed line in all figures represents the time at which the moving background is replaced by darkness.

nificantly correlated for 2 subjects both before and after stimulus termination, but are not significantly correlated for the third subject (T.H.). Table 1 also presents correlations for results averaged across subjects at each sampled time point. These analyses indicate that IM magnitude and ASA location are significantly correlated both before and after background motion and with both background velocities. ${ }^{1}$

Figures 1 and 2 permit visual comparison of ASA location with IM magnitude. Because we were interested in testing whether IM is caused by opposite ASA motion, ASA location results were converted to velocity measures. This was accomplished by subtracting the value of each ASA location sample from the succeeding sample and dividing the difference by 5 (the number of seconds between samples). The ASA velocities determined in this manner are plotted in Figures 3 and 4 for background velocities of 5 and $60 \mathrm{dps}$, respectively. The IM measures are again plotted in these figures to allow visual comparison with ASA velocity. It is apparent in the figures that, during stimulation, ASA moves initially in the same direction as background motion. However, after IM attains its maximum asymptotic magnitude, ASA velocity fluctuates around zero.

Correlations between IM and ASA velocity were computed in the same manner as that described earlier for correlations between IM and ASA location. These analyses indicate no significant relationship between IM magnitude and ASA velocity for any subject during background motion of either 5 or $60 \mathrm{dps}$. After background termination, IM magnitude and ASA velocity either do not correlate significantly or have significant negative 

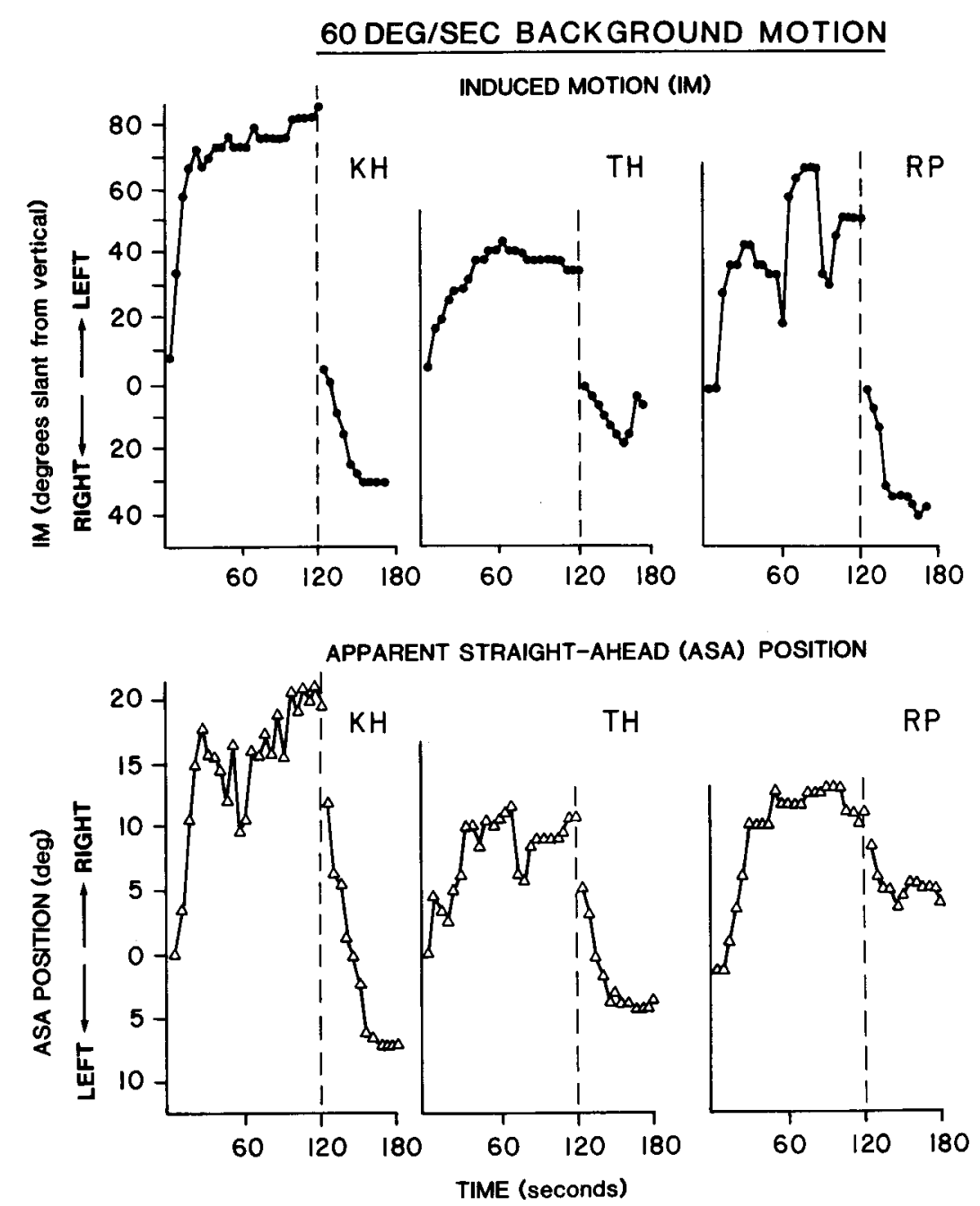

Figure 2. Top panel: Induced motion magnitude for individual subjects as a function of time both during and after presentaton of a 60-dps moving background. Bottom panel: Apparent straight-ahead position during the same time intervals for individual subjects.

correlations. These negative correlations indicate ASA movement in the same direction as IM, not the opposite direction. A summary of the correlations is presented in Table 2 .

\section{DISCUSSION}

IM magnitude increases and then asymptotes at a constant value during prolonged exposure to a moving background. Change in ASA location follows a similar time course, moving away from the objective midline to a stable displaced location. Following termination of the moving background, both IM magnitude and ASA location decay toward their prestimulation levels. Comparison of ASA velocity with IM magnitude reveals either no correlation or a negative correlation between these variables. Negative correlations of IM magnitude and ASA velocity indicate ASA movement in the same direction as IM, not the opposite.
This pattern of results is inconsistent with the hypothesis that IM results from ASA movement in the opposite direction (see, e.g., Bridgeman \& Klassen, 1983; Brosgole, 1968). If IM results from opposite motion of the ASA, then large values of IM should be observed when large motions of ASA are also occurring. Conversely, large IM magnitudes should not occur when ASA location is relatively stable. The present results do not confirm these predictions. Rather, IM magnitude was greatest

Table 1

Correlations of IM Magnitude with ASA Position Across 5-sec Time Samples for Individual Subjects

\begin{tabular}{|c|c|c|c|c|c|c|c|c|}
\hline \multirow{2}{*}{$\begin{array}{l}\text { Moving } \\
\text { Contour } \\
\text { Velocity }\end{array}$} & \multicolumn{4}{|c|}{$\begin{array}{c}\text { Before Stimulus } \\
\text { Termination }(d f=23)\end{array}$} & \multicolumn{4}{|c|}{$\begin{array}{c}\text { After Stimulus } \\
\text { Termination }(d f=9)\end{array}$} \\
\hline & K.H. & T.H. & R.P. & Group & K.H. & T.H. & R.P. & Group \\
\hline $\begin{array}{l}5 \mathrm{dps} \\
60 \mathrm{dps}\end{array}$ & $\begin{array}{l}.846 \dagger \\
.875 \dagger\end{array}$ & $\begin{array}{l}.301 \\
.825+\end{array}$ & $\begin{array}{l}.658 \\
.690 \dagger\end{array}$ & $\begin{array}{l}.839 \dagger \\
.919 \dagger\end{array}$ & $\begin{array}{l}.981 \dagger \\
.967 \dagger\end{array}$ & $\begin{array}{l}.494 \\
.645^{*}\end{array}$ & $\begin{array}{l}.821 \dagger \\
.701^{*}\end{array}$ & $\begin{array}{l}.941 \dagger \\
.970 \dagger\end{array}$ \\
\hline
\end{tabular}


INDUCED MOTION (IM)
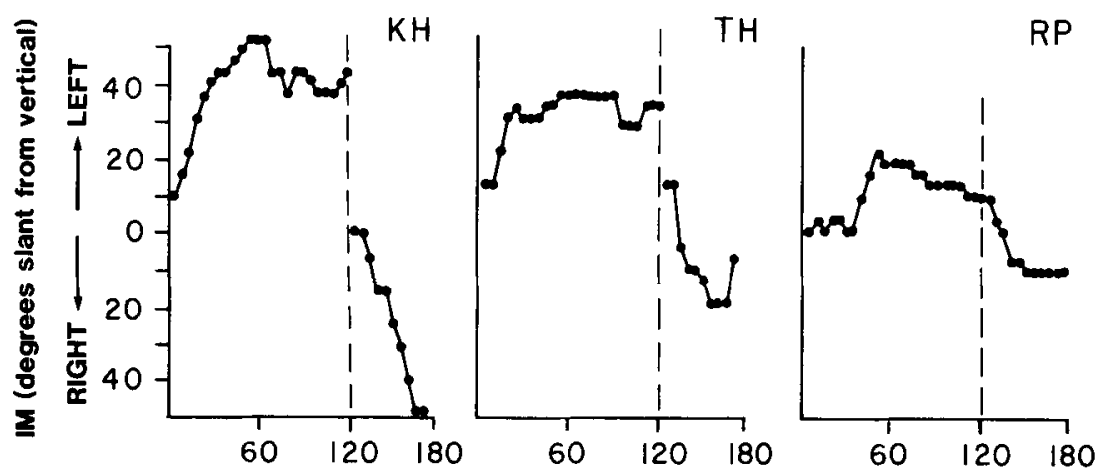

APPARENT STRAIGHT-AHEAD (ASA) MOTION

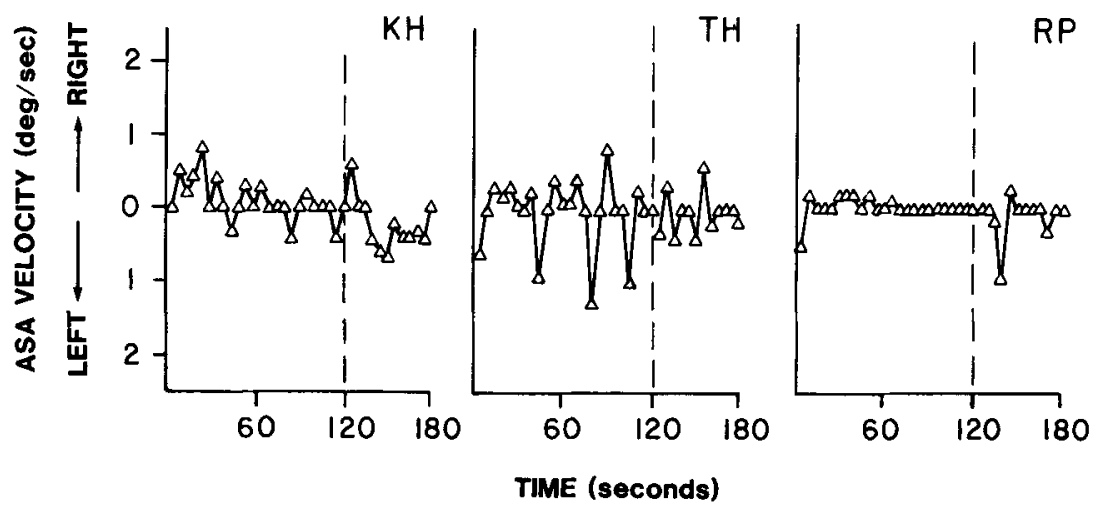

Figure 3. Top panel: IM magnitude for individual subjects across 5-sec time samples both during and after 5-dps moving background. Bottom panel: ASA velocity during the same time intervals for individual subjects.

when ASA was essentially stationary. Therefore, ASA movement cannot have caused the IM observed in the present study.

The present results are, however, consistent with an oculomotor account of IM. In particular, it has been proposed that use of the voluntary ocular pursuit system to suppress reflexive eye movements while viewing an IM display contributes to the illusion (Post, in press; Post et al., 1984). It is asserted that the moving background activates a reflexive eye-movement tendency which must be opposed if fixation is to be maintained. Illusory motion of the fixated target occurs because effort in the pursuit system is registered as motion of the fixated object. The present finding that IM magnitude increases gradually during background motion is consistent with the eyemovement suppression hypothesis because reflexive eyemovement activation is known to increase with stimulus duration (ter Braak, 1981; for a thorough review see Baloh et al., 1982). To summarize, while subjects view the IM display, activation in the reflexive eye-movement system gradually increases. Pursuit effort required to sup- press this activation similarly increases, and with it, IM magnitude.

Another major category of explanations for IM emphasizes the role of relative motion of elements within the visual display. It is not apparent that such explanations would predict the gradual increases in IM magnitude obtained during background motion in the present study, since the velocity of relative motion between the fixation target and the background is constant. Nor is it clear that such explanations would predict the pattern of effects obtained after moving background termination. Among these effects are: (1) for some subjects, continued illusory motion in the same direction as the preceding IM, (2) in all subjects, directional reversal of illusory motion, and (3) gradual increase in the magnitude of the reversed illusory motion. Since there are no elements in relative motion following stimulus termination, accounts that emphasize relative motion cannot predict these effects.

The idea that IM is caused by suppression of reflexive eye movements is, however, able to predict the effects that follow background termination. It is well known that 

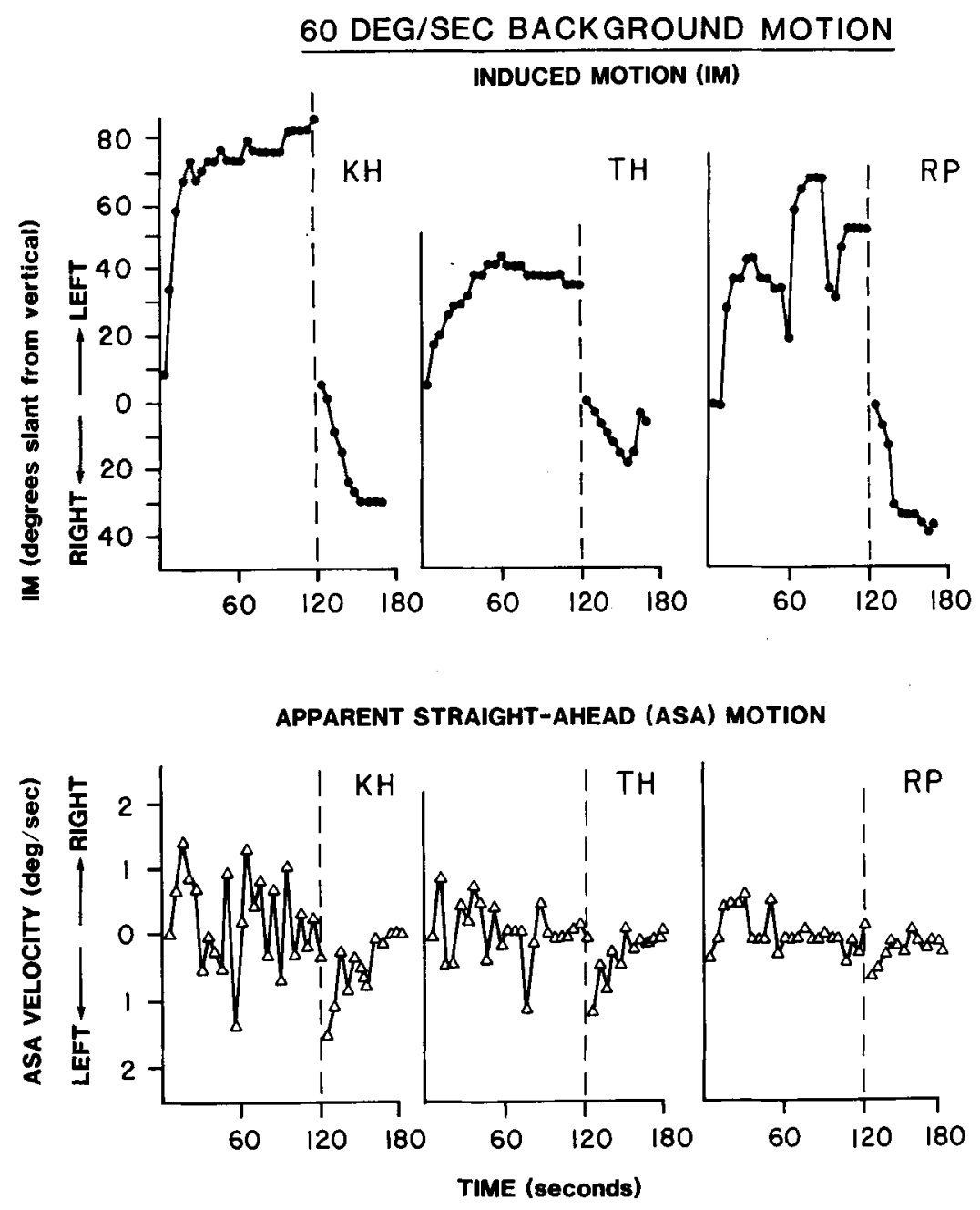

Figure 4. Top panel: IM magnitude for individual subjects as a function of time both during and after a 60-dps moving background. Bottom panel: ASA velocity during the same time intervals for individual subjects.

reflexive eye movements typically persist for some time following termination of a moving contoured display. Immediately after stimulus termination, such eye movements, termed optokinetic afternystagmus (OKAN), typically display slow-phase movements in the same direction as that of preceding contour motion (OKAN I). These movements are often followed by OKAN slow phase in the reverse direction (OKAN II) for some time before nystagmus fades altogether (Buchele, 1977). The known properties of OKAN are thus consistent with the present results if ocular pursuit activation suppresses reflexive eye movements to give illusory motion. Specifically: (1) continued illusory motion initially in the same direction as preceding IM is consistent with suppression by ocular pursuit of OKAN I; (2) directional reversal of illusory motion is consistent with suppression by ocular pursuit of OKAN II; and (3) increasing magnitude of illusory motion in the reverse direction is consistent with suppression by ocular pursuit of the typical gradual increase in OKAN II. Post (in press) has, in fact, reported that the direction and amount of poststimulus illusory motion correlates highly over a variety of stimulus conditions with the direction and amount of OKAN I and II in the manner predicted by the hypothesis of reflexive eyemovement suppression by ocular pursuit effort.

It is also of interest, within the context of this hypothesis, that robust IM was obtained in the present experiments with a 60-dps induction velocity. This result contrasts with others (e.g., Wallach \& Becklen, 1983), in which the effectiveness of moving contours in eliciting

Table 2

Correlations of IM Magnitude with ASA Velocity Across 5-sec Time Samples for Individual Subjects

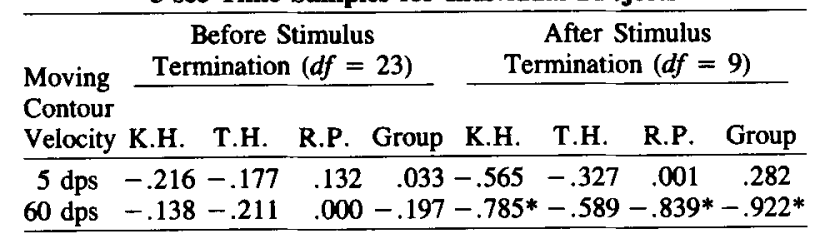

Note-dps $=$ degrees per second. ${ }^{*} p<.01$. 
IM rolls off at much lower velocities. It is likely that such differences in the literature arise from the variety of inducing stimuli that have been employed. For example, one common IM stimulus is a frame that moves slowly past a fixation target or oscillates back and forth about it (e.g., Bridgeman \& Klassen, 1983; Brosgole, 1968; Mack et al., 1985). It is clear that such slowly moving contours in an otherwise empty visual field are capable of eliciting reflexive gaze responses (Cheng \& Outerbridge, 1975) which are correlated with IM of opposite directional sign, as predicted by the present oculomotor theory (Post et al., 1984). However, since such an inducer can move only a limited distance before it must be extinguished or reversed, it must move either too slowly or too briefly to optimally activate optokinetic mechanisms, according to their known response characteristics (see Baloh et al., 1982, for a review). Failure of substantial reflexive gaze activation with brief, rapid frame movements possibly explains the upper velocity rolloff obtained with moving frame stimuli. Alernatively, the prolonged, repetitive, high-velocity, large-field induction stimulus used in the present experiments is known to be particularly effective in eliciting reflexive gaze activation. Also, the increase in IM magnitude from 5- to 60-dps stimulation is consistent with the stimulus velocity dependency displayed by optokinetic responses. In fact, Post (in press) obtained parallel increases in OKAN and IM with an increase in stimulus velocity from about 7 to $60 \mathrm{dps}$.

If IM is caused by ocular pursuit effort to maintain fixation opposite reflexive gaze activation, then the position constancy signal associated with ocular pursuit effort must also be active during IM (Heckmann, 1986). This position constancy signal possibly explains the ASA positional deviation associated with IM in the present as well as previous experiments (e.g., Brosgole, 1968; Mack et al., 1985). Specifically, the position constancy mechanism behaves like a leaky integrator with respect to the velocity information present in pursuit effort such that, at any given moment, ASA position is displaced opposite and in proportion to pursuit effort. During actual pursuit eye movements, ASA thus remains more or less aligned with the objective median plane. However, if pursuit effort is utilized to maintain steady fixation, as during IM, ASA is displaced from the objective median plane in a direction opposite to that of the pursuit effort. The present findings of ASA positional displacement may be regarded as analogous to those of Skavenski et al. (1972), who obtained ASA displacements proportional to, and in the same direction as, mechanical forces applied to the eye but resisted by fixation on a stationary target. In the present experiments, a reflexive tendency toward eye displacement replaces a mechanical one as the force that must be resisted by fixational effort.

The exact nature of the ASA shift obtained by us and by others (e.g., Brosgole, 1968; Mack et al., 1985) in connection with IM deserves some comment. Harris (1974) has distinguished ASA shifts that are "cognitive or conceptual," being judgments made with reference to visual detail, from "perceptual" ASA shifts, that is, "true" shifts of the subjective egocenter. The latter are defined by Harris as ASA shifts measurable in the absence of reference detail. Harris also discusses the difficulty of distinguishing between the two types of ASA shift, particularly when they are visually induced, as during IM (see, e.g., Mack et al., 1985). The present experiments, however, provided conditions that, by Harris's criteria, may allow at least part of the ASA shift associated with IM to be interpreted as "perceptual." We refer to the finding that IM magnitude and ASA deviation are dynamically correlated in the dark for at least a minute following termination of the induction stimulus; that is, an ASA shift occurred which was temporally distant from the visual reference detail provided by the induction stimulus. Moreover, the strong correlation of ASA position with IM magnitude under this condition, as well as during induction, suggests that IM and ASA are related to one another through the same mechanism under both conditions. Regardless of the particular category of ASA shift obtained in the present experiments, the lack of a relationship between IM and ASA velocity remains inconsistent with a theory that posits a causal role for ASA motion in IM.

To summarize, the present data suggest that IM is not caused by opposite ASA motion, since ASA velocity and IM magnitude are not related in the manner required by such a causal account. Furthermore, a relative-motion explanation of IM cannot predict the pattern of aftereffects presently reported. Gradual increase in IM magnitude during prolonged stimulation is, however, consistent with an eye-movement suppression hypothesis for IM, since reflexive eye-movement activation typically also increases gradually. The pattern of illusory motion following stimulus termination is also consistent with suppression of reflexive eye movements, which tend to reverse after stimulus termination in the absence of a fixation target.

We do not intend to suggest that ASA motion never contributes to IM, but, rather, that such motion is not able to account for the present results. Similarly, we do not believe that relative motion is unimportant for IM; rather, it does not predict the pattern of results reported here. We agree with Nakayama and Tyler (1978) that IM can probably be best accounted for by a combination of factors. Nakayama and Tyler's finding of simultaneous IM in opposite directions provides strong evidence for the contribution of relative motion. Other results, such as the gradual buildup in IM magnitude reported here or the reversal of both OKAN and illusory motion reported after removal of the moving background (Post, in press), provide evidence for the contribution of oculomotor factors.

\section{REFERENCES}

Baloh, R. W., Yee, R. D., \& Hornrubia, V. (1982). Abnormalities of optokinetic nystagmus. In G. Lennerstrand, D. S. Zee, \& E. L. Koller (Eds.), Functional basis of oculomotor disorders. Oxford: Pergamon. 
Brecher, G. A., Brecher, M. H., Kommerell, G., Sauter, F. A., \& SELLERBECK, J. (1972). Relation of optical and labyrinthean orientation. Optica Acta, 19, 467-471.

Bridgeman, B., \& KLASSEN, H. (1983). On the origin of stroboscopic induced motion. Perception \& Psychophysics, 34, 149-154.

Brosgole, L. (1968). Analysis of induced motion. Acta Psychologica, 28, $1-44$

BUCHELE, W. (1977). Optokinetischer Nachnystagmus und Bewegungshabituation beim Menschen in Abhangigkeit von der Reizdauer. Inauguraldissertation in medicine, at University of Freiburg, West Germany.

Cheng, M., \& Outerbridge, J. S. (1975). Optokinetic nystagmus during selective retinal stimulation. Experimental Brain Research, 23 129-139.

DUNCKER, K. (1929). Über induzierte Bewegung. Psychologische Forschung, 12, 180-259.

Gogel, W., \& MacCracken, P. J. (1979). Depth adjacency and induced motion. Perceptual \& Motor Skills, 48, 343-350.

HARRIs, C. S. (1974). Beware of the straight-ahead shift-a nonperceptual change in experiments on adaptation to displaced vision. Perception, 3, 461-476.

HeckmanN, T. (1986). Induced motion and orienting responses: $A$ new model of relationships among OKAN, induced motion, egocentric orientation and vection. Unpublished doctoral dissertation, University of California, Davis.

Mack, A., Heuer, F., Fendrich, R., Vilardi, K., \& Chambers, D. (1985). Induced motion and oculomotor capture. Journal of Experimen tal Psychology: Human Perception \& Performance, 11, 329-345.

NAKAYAMA, K., \& TYLER, C. W. (1978). Relative motion induced between stationary lines. Vision Research, 18, 1663-1668.
Post, R. B. (in press). Induced motion considered as a visually induced oculogyral illusion. Perception.

Post, R., Leibowitz, H. W. (1985). A revised analysis of the role of efference in motion perception. Perception, 14, 631-643.

Post, R., Shupert, C., \& LeIBowITz, H. (1984). Implications of OKN suppression by smooth pursuit for induced motion. Perception \& Psychophysics, 36, 493-498.

Skavenski, A. A., Haddad, G., \& Steinman, R. M. (1972). The extraretinal signal for the visual direction of perception. Perception \& Psychophysics, 11, 287-290.

TER BRAAK, J. W. G. (1981). Investigations on optokinetic nystagmus. In $\mathrm{H}$. Collewijn (Ed.), The oculomotor system of the rabbit and its plasticity. Berlin: Springer. (Original work published 1936 in German).

Wallach, H., Bacon, J., \& Schulman, P. (1978). Adaptation in motion perception: Alteration of induced motion. Perception \& Psychophysics, 24, 509-514.

WALLACH, H., \& BECKLEN, R. (1983). An effect of speed on induced motion. Perception \& Psychophysics, 34, 237-242.

\section{NOTE}

1. It may be argued that these analyses should be performed on the horizontal velocity vector component of IM rather than the slant angle. When this is done, however, the pattern of results reported is not influenced, because the tangent function of slant (which yields the horizontal velocity component) is effectively linear with respect to slant for most of the range of slants obtained.

(Manuscript received November 18, 1985; revision accepted for publication August 5, 1986.) 